\title{
Childhood maltreatment and DSM-IV adult mental disorders: comparison of prospective and retrospective findings
}

\author{
Kate M. Scott, Katie A. McLaughlin, Don A. R. Smith and Pete M. Ellis
}

\section{Background}

Prior research reports stronger associations between childhood maltreatment and adult psychopathology when maltreatment is assessed retrospectively compared with prospectively, casting doubt on the mental health risk conferred by maltreatment and on the validity of retrospective reports.

\section{Aims}

To investigate associations of psychopathology with prospective $v$. retrospective maltreatment ascertainment.

\section{Method}

A nationally representative sample of respondents aged 16-27 years $(n=1413)$ in New Zealand completed a retrospective assessment of maltreatment and DSM-IV mental disorders. Survey data were linked with a national child protection database to identify respondents with maltreatment records (prospective ascertainment).

\section{Results}

Childhood maltreatment was associated with elevated odds of mood, anxiety and drug disorders (odds ratios $=2.1-4.1$ ), with no difference in association strength between prospective and retrospective groups. Prospectively ascertained maltreatment predicted unfavourable depression course involving early onset, chronicity and impairment.

\section{Conclusions}

Prospectively and retrospectively assessed maltreatment elevated the risk of psychopathology to a similar degree. Prospectively ascertained maltreatment predicted a more unfavourable depression course.

\section{Declaration of interest}

None.
Although the mental health consequences of childhood maltreatment are thought to be considerable, ${ }^{1-3}$ determining the strength of this association has proved problematic because prospective ascertainment of childhood abuse and neglect is difficult. ${ }^{4,5}$ The few studies with prospective ascertainment of abuse are inconsistent, with some documenting an association with psychopathology ${ }^{6-8}$ and others suggesting a weak or non-existent relationship. ${ }^{9,10}$ A recent major review concluded that studies with retrospective assessment of maltreatment provided substantially stronger evidence for an association between childhood maltreatment and subsequent mental ill health than studies with prospective ascertainment of maltreatment. ${ }^{2}$ This is concerning, because doubts have been cast on the validity and reliability of retrospective accounts of childhood maltreatment, ${ }^{11-14}$ particularly from experimental research indicating the reconstructive nature of memory, ${ }^{15,16}$ and its susceptibility to mood congruent recall bias. ${ }^{17,18}$

We recently conducted a study that linked New Zealand national child protection agency records with the data from a nationally representative community survey of DSM-IV mental disorders to identify which survey respondents had a child protection agency history. We found that prospectively ascertained maltreatment in childhood (i.e. child protection agency history) was associated with a wide range of lifetime and past-year mood, anxiety and substance use disorders assessed in young adulthood. ${ }^{4}$ This paper examines these data further to compare prospective ascertainment with retrospective report of several major types of maltreatment in childhood (rape and sexual abuse, physical abuse, and witnessing inter-parental violence). To the best of our knowledge, this is only the second such study of its kind, and the first one to be based on a general population sample.

Our aims were twofold. First, to determine whether these data replicate the pattern observed in prior research ${ }^{10}$ of a stronger association between retrospectively, than prospectively, ascertained maltreatment and mental disorders. If this pattern is observed, it would bolster doubts about the true magnitude of long-term mental health harm conferred by childhood maltreatment (and about the validity of retrospective reports of maltreatment). Second, we aimed to provide the first known data on the association between prospectively ascertained childhood maltreatment and depressive disorder course.

We include the two disorders that have shown the greatest discrepancy in associations between retrospectively $v$. prospectively ascertained maltreatment: major depression ${ }^{9}$ and drug use disorder, ${ }^{10}$ and we also examine alcohol use disorder and anxiety disorders to provide a more comprehensive perspective.

\section{Method}

\section{Sample}

The New Zealand Mental Health Survey (NZMHS) was a nationally representative community survey involving face-to-face interviews with 12992 adults aged 16 and older conducted in 2003-2004 with a response rate of $73.3 \%$. Participants gave written informed consent. Ethics approval was obtained from the 14 New Zealand regional ethics committees. Internal subsampling was used to reduce respondent burden by dividing the interview into two parts. Part 1 included the core diagnostic assessment of mood disorders, alcohol use disorders and most of the anxiety disorders. Part 2 assessed the remainder of mental disorders and disorder correlates, including retrospective assessment of childhood maltreatment. All respondents completed Part 1. All Part 1 respondents who met criteria for any mental 
disorder and a probability sample of other respondents completed Part 2. These respondents $(n=7435)$ were weighted by the inverse of their probability of selection for Part 2 to adjust for differential sampling. This paper includes respondents aged 16-27 in the Part 2 subsample $(n=1413)$. The survey methods are described in detail elsewhere. ${ }^{19}$

\section{Measures}

\section{Mental disorders}

The World Mental Health version of the World Health Organization (WHO) Composite International Diagnostic Interview (CIDI 3.0), ${ }^{20}$ is a fully structured lay-administered interview that ascertains lifetime and past-year prevalence of disorder as well as recency of episodes. Disorders were assessed using DSM-IV criteria. ${ }^{21}$ The disorders included here are: major depressive disorder; any anxiety disorder (panic disorder with or without agoraphobia, specific phobia, social phobia, generalised anxiety disorder, post-traumatic stress disorder, obsessivecompulsive disorder); alcohol use disorders (abuse and dependence); and drug use disorders (abuse and dependence). Diagnoses were made using DSM-IV hierarchy rules.

\section{Functional impairment}

Respondents with symptoms of major depressive disorder in the past year were administered the disorder-specific Sheehan Disability Scale, ${ }^{22}$ which assesses functional impairment due to depression in the worst month of the past year in four domains: work, household, close relationships and social roles. Responses were providing on a $0-10$ visual analogue scale, with response options labelled none (0), mild (1-3), moderate (4-6), severe (7-9) and very severe (10). In addition, respondents were asked to estimate the number of days in the past 365 days when they were 'totally unable to work or carry out your normal activities' because of depression.

\section{Retrospectively ascertained childhood maltreatment}

This was based on retrospective assessment in the NZMHS of three of the five accepted dimensions of childhood maltreatment: ${ }^{2}$ physical abuse, sexual abuse and witnessing intimate partner violence. Sexual abuse was measured in terms of penetrative abuse (referred to here as childhood rape) or other non-penetrative abusive contact occurring prior to age 17 (referred to here as childhood sexual abuse). Respondents were coded as positive for sexual abuse if they endorsed either penetrative abuse or unwanted sexual touching or sexual assault and indicated that it had occurred prior to age 17. Childhood physical abuse was assessed with a question about being badly beaten up by parents or other caregivers. Participants were coded positive for physical abuse if they endorsed the item. Witnessing parental violence was assessed with a question about witnessing serious physical fights at home, such as one parent beating another. Participants were coded positive for witnessing violence if they endorsed the item. (See Scott et al for more detail about the questions.)

\section{Prospectively ascertained maltreatment}

Survey respondents were coded positively if they were identified as having been a primary client, beyond the initial assessment, in the electronic database of New Zealand's national child protection agency Child, Youth and Family (CYF). Although there are potential limitations to this data, it is reasonable to assume that this level of statutory child protection involvement with a child is an adequate proxy measure for the existence of at least one form of the five accepted dimensions of childhood maltreatment.

Further detail on the notification process, and on the method of linking the CYF database with the mental health survey database in order to identify matches, is provided in our earlier paper. ${ }^{4}$ Ethics approval for the data linkage and subsequent analysis was obtained from the New Zealand Multi-region Ethics committee.

The median time between the age at which the CYF file was opened and the age of mental disorder assessment was 10 years. Because the national electronic CYF database commenced in 1990 , we examine only survey respondents who were aged 1627 when interviewed (age range $3-13$ in 1990). A total of $10 \%$ of these respondents were identified as having a CYF history. This proportion is high by international standards and may reflect a substantively higher rate of childhood maltreatment in New Zealand $^{23}$ and/or possibly a lower threshold for notification due to the high public profile of childhood maltreatment. Agency involvement was lower (5-6\%) for respondents aged 26-27, which may indicate CYF involvement prior to 1990 for this age group that was not captured in the electronic database.

\section{Sociodemographic correlates}

Ethnicity included the indigenous Maori group, the Pacific group (those emigrating or originating from Pacific Island nations) and all others. Additional correlates included age at interview, gender, maternal education, respondent's educational attainment and respondent's household income (adjusted for household size) ${ }^{24}$ at the time of the survey. Item non-response was minimal, with two exceptions. Household income was missing for $13 \%$ of the survey sample. Missing values were imputed by multiple regression with weights using age, gender, education, marital status, employment status, current or previous job held, time since last employed, number in the household, and the New Zealand Index of Deprivation. ${ }^{19}$ Maternal education was missing for $3 \%$ of 16 - to 27 -year-olds, but for $9 \%$ of those with child protection agency history (Table 1); 'missing' was therefore coded as a level on the maternal education variable to adjust for this disparity.

\section{Statistical analysis}

Estimates were weighted to adjust for selection probabilities, nonresponse, intentional oversampling of Maori and Pacific peoples and post-stratified to match age, gender and ethnicity distributions to the 2001 census population. Cross-tabulations provided mental disorder prevalence. Multivariable logistic regression analyses, with and without adjustment for sociodemographic covariates, estimated odds of mental disorders among the three study groups: (a) those with prospectively ascertained maltreatment (i.e. all those with child protection agency history regardless of whether they also reported it retrospectively); (b) those with retrospectively ascertained maltreatment (i.e. those survey respondents without a child protection agency history who reported maltreatment in childhood); and (c) those with no reported maltreatment (all others: this group served as the comparison group in regression analyses). Although a substantial proportion (45\%) of the prospective group also retrospectively reported maltreatment, the prospective group is distinguished from the retrospective group by their history of child protection agency contact, meaning that any association between this group and subsequent mental disorders cannot be attributed to recall biases. 
Table 1 New Zealand Mental Health Survey sample characteristics for persons with and without a history of childhood maltreatment, ages $16-27^{\mathrm{a}}$

Prospectively ascertained Retrospectively ascertained No reported maltreatment maltreatment in childhood ${ }^{\mathrm{b}} \quad$ maltreatment in childhood ${ }^{\mathrm{c}}$ in childhood

\begin{tabular}{|c|c|c|c|}
\hline Unweighted $n$ in total sample $(n=2144)$ & 221 & 358 & 1565 \\
\hline Unweighted $n$ in Part 2 subsample $(n=1413)$ & 168 & 358 & 887 \\
\hline Age, years: mean & 21 & 22 & 21 \\
\hline \multicolumn{4}{|l|}{ Ethnicity, \% (n) } \\
\hline Maori & $38.0(86)$ & $28.3(127)$ & $13.8(199)$ \\
\hline Pacific & $6.5(37)$ & $13.2(130)$ & $6.0(202)$ \\
\hline Other & $55.6(45)$ & $58.5(101)$ & $80.2(486)$ \\
\hline Female, \% (n) & $64.6(113)$ & $60.6(230)$ & $48.2(471)$ \\
\hline \multicolumn{4}{|l|}{ Educational qualifications, \% (n) } \\
\hline None & $34.5(64)$ & $22.3(91)$ & $10.0(134)$ \\
\hline Secondary school or post-secondary school & $43.7(75)$ & $47.7(175)$ & $62.9(495)$ \\
\hline Both secondary school and post-secondary school & $21.8(29)$ & $30.1(92)$ & $27.1(258)$ \\
\hline \multicolumn{4}{|l|}{ Equivalised household income, \% (n) } \\
\hline Under half of median & $40.7(84)$ & 33.4 (139) & $24.0(243)$ \\
\hline Half median to median & $37.5(53)$ & $37.1(127)$ & $31.9(297)$ \\
\hline Median to 1.5 times median & $14.2(24)$ & $19.0(62)$ & $26.8(219)$ \\
\hline 1.5 times median and over & $7.8(7)$ & $10.6(30)$ & $17.3(128)$ \\
\hline \multicolumn{4}{|l|}{ Mother's education, \% ( $n$ ) } \\
\hline Primary & $12.6(22)$ & $8.8(53)$ & $3.6(65)$ \\
\hline Secondary & $56.0(101)$ & $61.1(217)$ & $54.0(499)$ \\
\hline Tertiary & $23.2(32)$ & $27.0(71)$ & 40.7 (299) \\
\hline Don't know/missing & $8.2(13)$ & $3.2(17)$ & $1.7(24)$ \\
\hline
\end{tabular}

The remaining analyses concerned major depressive disorder only. The percentage reporting severe or very severe $(7+)$ impairment associated with major depressive disorder was derived for each of the three groups from logistic regression models generating multivariate-adjusted percentages (predicted marginals). These models were initially run adjusting for socioeconomic correlates, but as none of these correlates were significant in the models, the results shown are from models without the correlates included. Predicted marginals from non-linear models are analogous to adjusted group means in linear regression and $t$-tests were used to examine pairwise comparisons (i.e. the general linear contrast of the marginals). Linear regression models estimated the mean days out of role for the three groups. Taylor series linearisation ${ }^{25}$ was used to approximate the variance of estimates using SAS-callable SUDAAN 10.0.1 (Windows XP) to adjust for the complex sampling design.

\section{Results}

\section{Sample characteristics}

Sample characteristics for the three groups are provided in Table 1. The two groups with maltreatment history had lower socioeconomic status (in terms of respondent educational attainment,

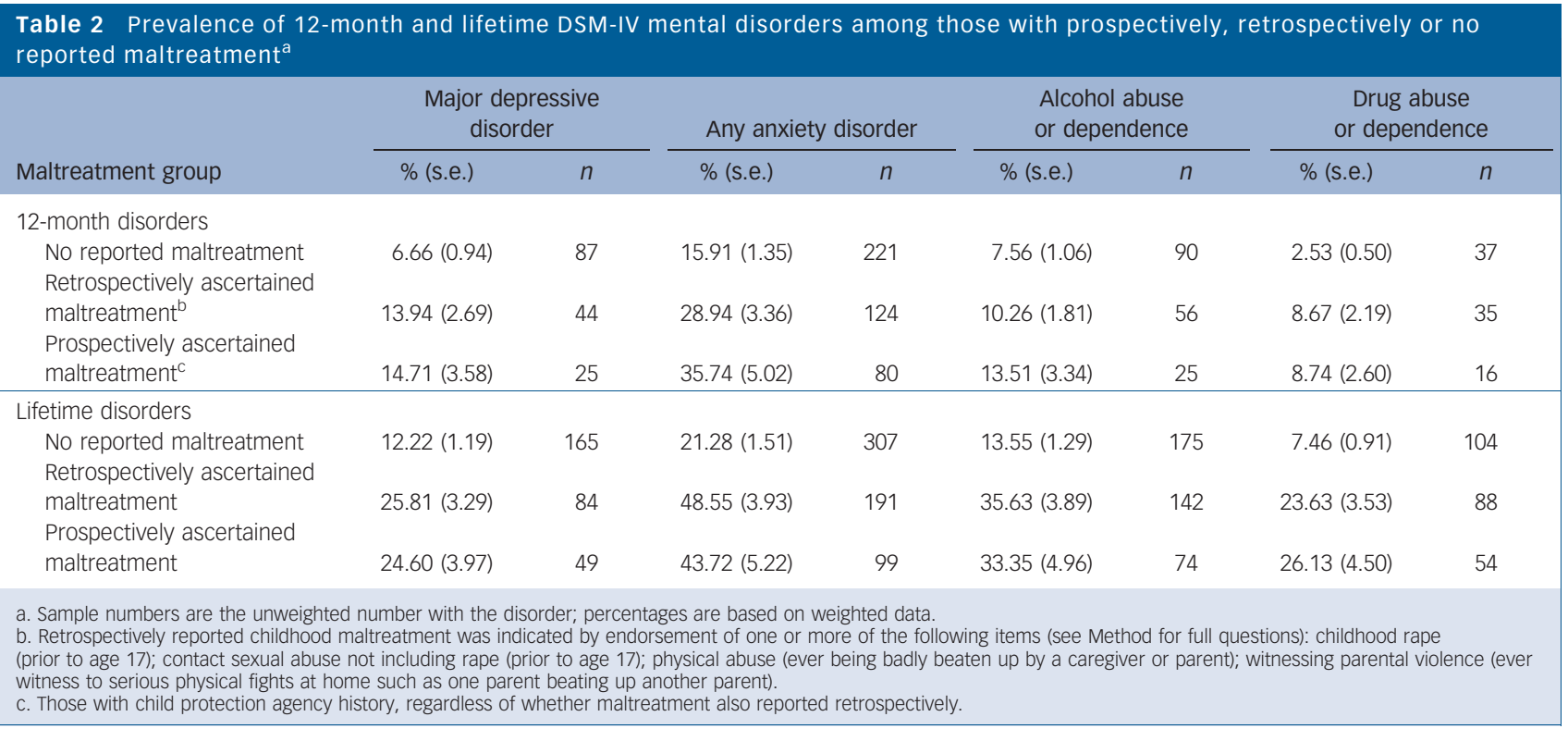




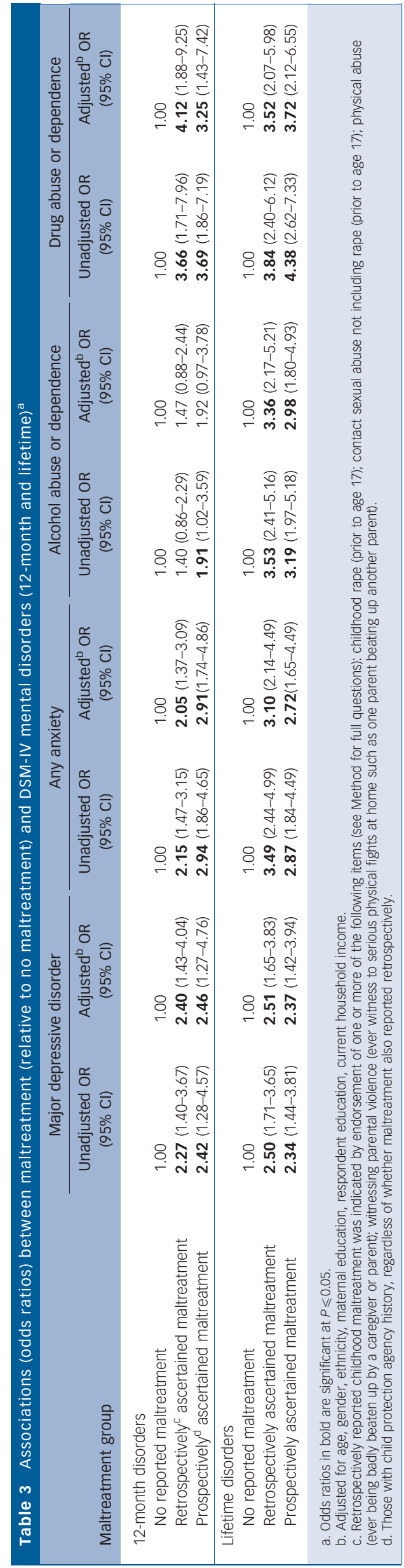

mother's education, respondent household income) relative to the group with no maltreatment. Of the two maltreatment groups, the group with prospective ascertainment was the more disadvantaged. Both maltreatment groups had a higher proportion of females and indigenous Maori people relative to the comparison group.

\section{Disorder prevalence}

Table 2 provides the prevalence of mental disorder among the three groups. Respondents with a history of maltreatment have a higher prevalence of mental disorders relative to those with no maltreatment history, but there are not pronounced differences in disorder prevalence between the two maltreatment groups. Importantly, these data are descriptive, without adjustment for sociodemographic characteristics.

\section{Associations of maltreatment with mental disorder}

Results of the logistic regression analyses shown in Table 3 indicate that maltreatment is significantly associated with every 12 -month disorder examined, with the exception of alcohol use disorder, after adjustment for sociodemographics. Odds of a 12-month mental disorder are significantly elevated two- to fourfold in both maltreatment groups relative to those without reported maltreatment. The strength of associations between prospectively and retrospectively ascertained maltreatment and 12-month mental disorders is similar across all outcomes.

The associations between childhood maltreatment and lifetime disorders follow the same pattern observed for 12-month disorders, with adjusted odds ratios in the range of 2.4-3.7 associated with exposure to maltreatment. The magnitude of associations does not differ across the two maltreatment groups.

\section{Associations of maltreatment with major depressive disorder onset and course}

Table 4 shows the age at onset distribution of major depressive disorder and the distribution of number of lifetime episodes in each maltreatment group. This analysis indicates the youngest median age at onset for individuals with prospectively ascertained maltreatment (14 years, interquartile range $(\mathrm{IQR})=12-17)$, followed by retrospectively assessed maltreatment (16 years, $\mathrm{IQR}=13-18)$ and no maltreatment controls $(18$ years, IQR $=15-$ 20). The prospectively ascertained maltreatment group also experienced a greater number of lifetime episodes of major depressive disorder (median 3.5, IQR $=1.5-10)$ than the retrospectively ascertained (median $2, \mathrm{IQR}=1-5$ ) and control groups (median 2, IQR = 1-4).

Finally, Table 5 provides information on the degree of functional impairment experienced by those with major depressive disorder in the three groups. Compared with the group

\begin{tabular}{|c|c|c|c|}
\hline & \multicolumn{3}{|c|}{ Percentile } \\
\hline & 25th & 50th & 75th \\
\hline \multicolumn{4}{|l|}{ Age at onset, years } \\
\hline No reported maltreatment & 15 & 18 & 20 \\
\hline Retrospectively ascertained maltreatment & 13 & 16 & 18 \\
\hline Prospectively ascertained maltreatment & 12 & 14 & 17 \\
\hline \multicolumn{4}{|l|}{ Lifetime episodes, $n$} \\
\hline No reported maltreatment & 1 & 2 & 4 \\
\hline Retrospectively ascertained maltreatment & 1 & 2 & 5 \\
\hline Prospectively ascertained maltreatment & 1.5 & 3.5 & 10 \\
\hline
\end{tabular}




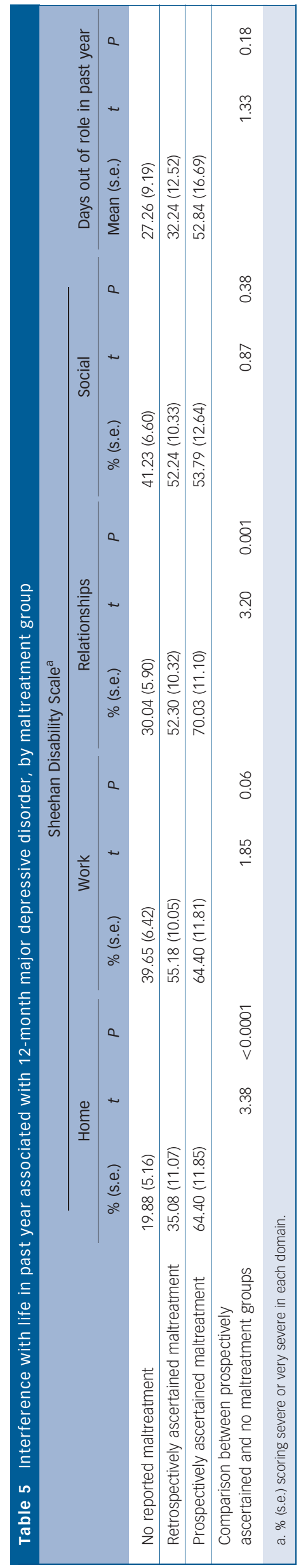

with no maltreatment history, the prospectively ascertained maltreatment group were significantly more likely to report severe impairment in two domains (home and relationships) and marginally more likely to report severe impairment in the work domain (contrasts shown in Table 5). Although the proportion reporting severe impairment was higher in the prospectively ascertained maltreatment group than the retrospective group for three out of four domains, these differences were of only marginal significance for the home domain $(t=1.81, P=0.07)$ and were not significant for the other domains. The prospective group also reported a greater number of days out of role than the retrospective group, although again this was not statistically significant $(t=0.98)$.

\section{Discussion}

\section{Main findings}

In this study we compared mental disorder outcomes between those with maltreatment histories that were prospectively ascertained (via child protection agency records) $v$. those that were retrospectively ascertained (via survey respondents' retrospective reports). We found that a history of maltreatment conferred a considerably higher risk of a range of mental disorders (mood, anxiety and drug use) in young adulthood, but there was no difference in the strength of associations as a function of whether maltreatment history was prospectively $v$. retrospectively ascertained. We also found that prospectively ascertained maltreatment predicted major depressive disorders of earlier onset, with greater frequency of episodes and with greater associated impairment. These findings contrast with the reports of the first major study on this topic that found associations between retrospectively ascertained maltreatment and mental disorders, but no associations, or much weaker associations, between prospectively ascertained maltreatment and mental disorders. 9,10 The implication of those earlier findings was that the associations between childhood maltreatment and at least some mental disorders were artefacts of recall bias. The results of our study, by contrast, provide no support for that conclusion. We found significant associations between prospectively ascertained childhood maltreatment and a range of subsequently assessed mental disorders, and major depressive disorder course; and we did not find that rates of mental disorders, either 12-month or lifetime, were higher among those with retrospectively ascertained maltreatment histories.

Prospective and retrospective accounts of childhood maltreatment do not necessarily yield information about the same type of risk. The prospective data tell us whether childhood abuse is a risk factor for subsequent poor health outcomes, and we believe our data, both here and from our previous study, ${ }^{4}$ confirm this. The retrospective data tell us whether childhood abuse, appraised and recalled as such by the individual, is a risk factor for subsequent poor outcomes. The nature of risk ascertained may not be identical across the two methodologies, but if it is not, we believe it is important to study both.

The final noteworthy feature of these results is that they constitute the first evidence of prospectively ascertained childhood maltreatment predicting a more unfavourable course of major depressive disorder. This is in agreement with the results of a recent review ${ }^{26}$ based on studies with retrospective assessment of maltreatment in childhood. Our findings of a somewhat earlier onset of depression, greater number of episodes and greater impairment in the prospective group suggest that their maltreatment began earlier in life and was possibly more severe (on average). This interpretation cannot be confirmed from these data but is consistent with our understanding of the often highly 
disadvantaged childhoods experienced by those with child protection agency contact, a disadvantage that can begin in the prenatal period.

\section{Findings from previous studies}

Amoung previous studies there were two in particular that elevated concern about relying on retrospective reports for our understanding of the links between childhood maltreatment and mental disorders. Widom and colleagues ${ }^{9}$ found no association between prospectively ascertained (court documented) childhood sexual abuse and major depressive disorder in adulthood, in contrast to the strong and consistent evidence for this association from retrospective studies. ${ }^{3,27,28}$ In another report from the same study, Widom and colleagues ${ }^{10}$ found a significant association between retrospectively, but not prospectively, ascertained childhood maltreatment and drug misuse in young adulthood. In a later review of this topic, Widom and colleagues ${ }^{29}$ concluded that recall bias can result in spurious associations between selfreported childhood maltreatment and adult mental health, thus challenging the widespread view, based on retrospective data, that childhood maltreatment contributes significantly to later mental disorders.

As we have previously suggested, ${ }^{4}$ this difference in findings may have arisen from Widom and colleagues ${ }^{10}$ using a control group drawn from the same low socioeconomic population as their court-documented maltreatment group. This avoided associations between maltreatment and later outcomes being confounded by social class, but resulted in a control group with very high rates $(49 \%)$ of childhood maltreatment. This would greatly diminish any differences between the two groups in terms of mental health outcomes. For example, Widom and colleagues reported that $11 \%$ of the court-documented maltreatment group met criteria for drug use disorder in adulthood, compared with $8 \%$ in the control group, an insignificant difference. ${ }^{10}$ In contrast, $12 \%$ of those retrospectively reporting childhood maltreatment had drug use disorder in adulthood, compared with $6 \%$ in those who did not report such abuse: a significant difference. Further, similar to the findings in the present study, there was a comparable prevalence of drug use disorder in the prospectively ascertained and the retrospectively ascertained maltreatment groups.

\section{Limitations}

This study has several limitations. One of the most important of these is the use of a proxy indicator of childhood maltreatment. We make no claim that national childhood protection agency history is as accurate an indicator of maltreatment as court documentation of abuse, but the extent of misclassification is likely to be small, and to the extent that it has occurred, it would serve to weaken associations, rendering the results we report here for the prospectively ascertained group conservative. It is also a limitation that the prospective and retrospective groups differ in a number of respects besides ascertainment method. They are drawn from different populations with different socioeconomic status. These differences were statistically adjusted for to the extent that our data allowed, but this may not have completely eliminated group differences in this respect. The groups may also differ in the nature and severity of maltreatment, as noted earlier, although this cannot be assumed to be the case. ${ }^{4}$ Finally, the retrospective group is based on assessment of physical and sexual abuse and the witnessing of family violence, but not neglect. This may mean that some neglected children would have been misclassified into the 'no maltreatment' group, thereby attenuating the associations between retrospectively reported maltreatment and psychopathology. Another important limitation is the possibility of sample selection bias (through those with child protection agency history being less willing to take part in the mental health survey, and those unwilling to take part in the survey being more likely to have mental disorders), but again this would mean that our findings probably underestimate the real strength of associations between childhood maltreatment and mental disorders.

In conclusion, this study provides rare population-based data comparing mental disorder outcomes among those with childhood maltreatment histories differentiated by prospective $v$. retrospective ascertainment of maltreatment. We found that associations between maltreatment and mental disorders are similar across both types of maltreatment ascertainment, which confirms the substantive risk conferred by childhood abuse for later mental health. Additionally, we have provided the first evidence of a link between prospectively ascertained childhood maltreatment and a more unfavourable course of depressive disorder.

\section{Kate M. Scott, PhD, Department of Psychological Medicine, Dunedin School of Medicine, University of Otago, Dunedin, New Zealand; Katie A. McLaughlin, PhD, Division of General Pediatrics, Children's Hospital Boston, Harvard Medical School, Boston, USA; Don A. R. Smith, MA, Pete M. Ellis, PhD, BM BCh, Department of} Psychological Medicine, University of Otago, Wellington, New Zealand

Correspondence: Kate M. Scott, PhD, Department of Psychological Medicine, Dunedin School of Medicine, University of Otago, PO Box 913, Dunedin 9054, New Zealand. Email: kate.scott@otago.ac.nz

First received 19 Sep 2011, final revision 19 Dec 2011, accepted 17 Jan 2012

\section{Funding}

Te Rau Hinengaro: The New Zealand Mental Health Survey (NZMHS) was funded by the Ministry of Health, Alcohol Advisory Council of New Zealand and Health Research Council of New Zealand. The survey was carried out in conjunction with the World Health Organization World Mental Health (WMH) Survey Initiative (WMH publications are listed at http://www.hcp.med.harvard.edu/wmh/). These activities were supported by the US National Institute of Mental Health (R01MH070884), the John D. and Catherine T. MacArthur Foundation, the Pfizer Foundation, the US Public Health Service (R13MH066849, R01-MH069864, and R01 DA016558), the Fogarty International Center (FIRCA R01-TW006481), the Pan American Health Organization, Eli Lilly and Company, Ortho-McNeil Pharmaceutical, GlaxoSmithKline and Bristol-Myers Squibb.

\section{Acknowledgements}

We thank the Kaitiaki Group and Pacific Advisory Group for their input and support for this survey and we thank all the participants. We thank the World Health Organization World Mental Health staff for assistance with instrumentation, fieldwork and data analysis. We thank the New Zealand Child Youth and Family agency for access to their electronic client database.

\section{References}

1 Kessler RC, McLaughlin KA, Green JG, Gruber MJ, Sampson NA, Zaslavsky $\mathrm{AM}$, et al. Childhood adversities and adult psychopathology in the WHO World Mental Health Surveys. Br J Psychiatry 2010; 197: 378-85.

2 Gilbert R, Widom CS, Browne K, Fergusson DM, Webb E, Janson S. Child maltreatment I. Burden and consequences of child maltreatment in highincome countries. Lancet 2009; 373: 68-81.

3 Green JG, Berglund PA, Gruber MJ, McLaughlin KA, Sampson NA, Zaslavsky $A M$, et al. Childhood adversities and adult psychopathology in the Nationa Comorbidity Survey Replication (NCS-R) I: associations with first onset of DSM-IV disorders. Arch Gen Psychiatry 2010; 67: 113-23.

4 Scott KM, Smith DR, Ellis PM. Prospectively ascertained child maltreatment and its association with DSM-IV mental disorders among young adults. Arch Gen Psychiatry 2010; 67: 712-9.

5 Hardt J, Rutter M. Validity of adult retrospective reports of adverse childhood experiences: review of the evidence. J Child Psychol Psychiatry 2004; 45 260-73. 
6 Trickett PK, Noll JG, Putnam FW. The impact of sexual abuse on female development: lessons from a multigenerational, longitudinal research study. Dev Psychopathol 2011; 23: 453-76.

7 Lansford JE, Dodge KA, Pettit GS, Bates JE, Crozier J, Kaplow J. A 12-year prospective study of the long-term effects of early child physical maltreatment on psychological, behavioral, and academic problems in adolescence. Arch Pediatr Adolesc Med 2002; 156: 824-30.

8 Widom CS. Postraumatic stress disorder in abused and neglected children grown up. Am J Psychiatry 1999; 156: 1223-9.

9 Widom CS, DuMont K, Czaja SJ. A prospective investigation of major depressive disorder and comorbidity in abused and neglected children grown up. Arch Gen Psychiatry 2007; 64: 49-56.

10 Widom CS, Weiler BL, Cottler LB. Childhood victimization and drug abuse: a comparison of prospective and retrospective findings. J Consult Clin Psychol 1999; 67: 867-80.

11 Fergusson DM, Horwood LJ, Woodward LJ. The stability of child abuse reports: a longitudinal study of young adults. Psychol Med 2000; 30: 529-44.

12 Widom CS, Morris S. Accuracy of adult recollections of childhood victimization: part 2. Childhood sexual abuse. Psychol Assess 1997; 9 : 34-46.

13 Williams LM. Recall of childhood trauma: a prospective study of women's memories of childhood sexual abuse. J Consult Clin Psychol 1994; 62: 1167-76.

14 Loftus EF, Garry M, Feldman J. Forgetting sexual trauma: what does it mean when 38\% forget? J Consult Clin Psychol 1994; 62: 1177-81.

15 Schacter DL, Koutstaal W, Norman KA. Can cognitive neuroscience illuminate the nature of traumatic childhood memories? Curr Opin Neurobiol 1996; 6: 207-14.

16 Ross M. Relation of implicit theories to the construction of personal histories. Psychol Rev 1989; 96: 341-57.

17 Blaney PH. Affect and memory: a review. Psychol Bull 1986; 99: 229-46.
18 Williams JMG, Watts FN, MacLeod C, Mathews A. Cognitive Psychology and Emotional Disorders (2nd edn). John Wiley and Sons, 1997.

19 Wells JE, Oakley Browne MA, Scott KM, McGee MA, Baxter J, Kokaua J. Te Rau Hinengaro: the New Zealand Mental Health Survey (NZMHS): overview of methods and findings. Aust N Z J Psychiatry 2006; 40: 835-44.

20 Kessler RC, Ustun B. The World Mental Health (WMH) Survey Initiative version of the World Health Organization (WHO) Composite International Diagnostic Interview (CIDI). Int J Method Psychiatr Res 2004; 13: 93-121.

21 American Psychiatric Association. Diagnostic and Statistical Manual of Mental Disorders (4th edn) (DSM-IV). APA, 1994.

22 Leon AC, Olfson M, Portera L, Farber L, Sheehan DV. Assessing psychiatric impairment in primary care with the in primary care with the Sheehan Disability Scale. Int J Psychiatr Med 1997; 27: 93-105.

23 OECD. Doing Better For Children. OECD, 2009 (http://www.oecd.org/els/ social/childwellbeing).

24 Jensen J. Income Equivalences and the Estimation of Family Expenditures on Children. Department of Social Welfare, 1988.

25 Shah BV. Linearization methods of variance estimation. In Encyclopedia of Biostatistics (eds P Armitage, T Colton): 2276-9. John Wiley and Sons, 1998.

26 Nanni V Uher R, Danese A. Childhood maltreatment predicts unfavourable course of illness and treatment outcome in depression: a meta-analysis. Am J Psychiatry 2012; 169: 141-51.

27 Mullen PE, Martin JL, Anderson JC, Romans SE, Herbison GP. Childhood sexual abuse and mental health in adult life. Br J Psychiatry 1993; 163: 721-32.

28 Kendler KS, Kuhn JW, Prescott CA. Childhood sexual abuse, stressful life events and risk for major depression in women. Psychol Med 2004; 34: 1475-82.

29 Widom CS, Raphael KG, DuMont K. The case for prospective longitudinal studies in child maltreatment research: commentary on Dube, Williamson, Thompson, Felitti and Anda (2004). Child Abuse Negl 2004; 28: $715-22$

Buchner compressed several lifetimes' worth of achievement into 23 years. By the time he died (of typhus), he was a university lecturer and he had written a ground-breaking dissertation On Cranial Nerves, the first revolutionary document in German history and two of the greatest plays ever written. Danton's Death was written in five weeks, during Buchner's flight into Switzerland from probable imprisonment. A gripping political drama, its insight into political intrigue is comparable to Shakespeare. Woyzeck, unfinished at Buchner's death, has inspired many of the key innovators in Western theatre. Rarely has such astounding promise been so cruelly cut short. 